Acta Crystallographica Section E

Structure Reports

Online

ISSN 1600-5368

\section{2-Methoxy-4-(prop-2-en-1-yl)phenyl benzoate}

\section{Mallikarjuna Rao Pichika, ${ }^{\mathrm{a} *}$ Yew Beng Kang ${ }^{\mathrm{a}}$ and Seik Weng $\mathbf{N g}^{\mathrm{b}, \mathrm{c}}$}

aDepartment of Pharmaceutical Chemistry, International Medical University, 126 Jalan Bukit Jalil, 57000 Kuala Lumpur, Malaysia, ${ }^{\mathbf{b}}$ Department of Chemistry, University of Malaya, 50603 Kuala Lumpur, Malaysia, and ${ }^{\mathrm{C} C h e m i s t r y ~ D e p a r t m e n t, ~}$ Faculty of Science, King Abdulaziz University, PO Box 80203 Jeddah, Saudi Arabia Correspondence e-mail: mallikarjunarao_pichika@imu.edu.my

Received 3 June 2013; accepted 6 June 2013

Key indicators: single-crystal X-ray study; $T=100 \mathrm{~K}$; mean $\sigma(\mathrm{C}-\mathrm{C})=0.002 \AA$; $R$ factor $=0.051 ; w R$ factor $=0.138 ;$ data-to-parameter ratio $=17.5$.

In the title compound, $\mathrm{C}_{17} \mathrm{H}_{16} \mathrm{O}_{3}$, the benzene rings are twisted by $63.54(5)^{\circ}$. The twist is similar to that found in the unsubstituted compound, phenyl benzoate. The crystal packing features $\mathrm{C}-\mathrm{H} \cdots \mathrm{O}$ hydrogen bonds.

\section{Related literature}

For the structure of phenyl benzoate, see: Shibakami \& Sekiya (1995).

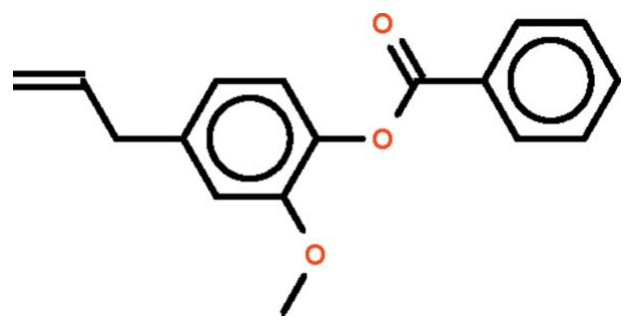

\section{Experimental}

Crystal data

$\mathrm{C}_{17} \mathrm{H}_{16} \mathrm{O}_{3}$

$M_{r}=268.30$

Monoclinic, $P 2_{1} / c$ $\beta=103.405(6)^{\circ}$

$V=1373.81(14) \AA^{3}$

$Z=4$

Mo $K \alpha$ radiation

Data collection

Agilent SuperNova Dual diffractometer with an Atlas detector

Absorption correction: multi-scan (CrysAlis PRO; Agilent, 2013)

$T_{\min }=0.966, T_{\max }=0.983$

Refinement

$R\left[F^{2}>2 \sigma\left(F^{2}\right)\right]=0.051$

$w R\left(F^{2}\right)=0.138$

$S=1.05$

3168 reflections

$\mu=0.09 \mathrm{~mm}^{-1}$

$T=100 \mathrm{~K}$

$0.40 \times 0.30 \times 0.20 \mathrm{~mm}$ 3168 independent reflections 2242 reflections with $I>2 \sigma(I)$ $R_{\text {int }}=0.041$

\section{Table 1}

Hydrogen-bond geometry $\left(\AA{ }^{\circ}\right)$.

\begin{tabular}{lllll}
\hline$D-\mathrm{H} \cdots A$ & $D-\mathrm{H}$ & $\mathrm{H} \cdots A$ & $D \cdots A$ & $D-\mathrm{H} \cdots A$ \\
\hline $\mathrm{C} 2-\mathrm{H} 2 \cdots \mathrm{O}^{\mathrm{i}}$ & 0.95 & 2.55 & $3.256(2)$ & 131 \\
$\mathrm{C} 15-\mathrm{H} 15 \cdots \mathrm{O}^{\mathrm{ii}}$ & 0.95 & 2.54 & $3.209(2)$ & 128
\end{tabular}

Symmetry codes: (i) $-x,-y+1,-z+1$; (ii) $x,-y+\frac{1}{2}, z+\frac{1}{2}$.

Data collection: CrysAlis PRO (Agilent, 2013); cell refinement: CrysAlis PRO; data reduction: CrysAlis PRO; program(s) used to solve structure: SHELXS97 (Sheldrick, 2008); program(s) used to refine structure: SHELXL97 (Sheldrick, 2008); molecular graphics: $X$-SEED (Barbour, 2001); software used to prepare material for publication: publCIF (Westrip, 2010).

We thank the Ministry of Higher Education of Malaysia (ERGS/1/2012/STG01/IMU/02/1; UM-C/HIR-MOHE/SC/03) for supporting this study and the International Medical University for providing the facilities.

Supplementary data and figures for this paper are available from the IUCr electronic archives (Reference: BT6914).

\section{References}

Agilent (2013). CrysAlis PRO. Agilent Technologies Inc., Santa Clara, CA, USA.

Barbour, L. J. (2001). J. Supramol. Chem. 1, 189-191.

Sheldrick, G. M. (2008). Acta Cryst. A64, 112-122.

Shibakami, M. \& Sekiya, A. (1995). Acta Cryst. C51, 326-330.

Westrip, S. P. (2010). J. Appl. Cryst. 43, 920-925. 


\section{supporting information}

Acta Cryst. (2013). E69, o1088 [https://doi.org/10.1107/S1600536813015791]

\section{2-Methoxy-4-(prop-2-en-1-yl)phenyl benzoate}

\section{Mallikarjuna Rao Pichika, Yew Beng Kang and Seik Weng Ng}

\section{S1. Comment}

The title phenyl benzoate (Scheme I, Fig. 1), which possesses an allyl and a methoxy substituent, was synthesized for an evaluation of its pharmaceutical properties as it is an ester derivative of eugenol. The two benzene rings are approximately perpendicular [dihedral angle $63.54(5)^{\circ}$ ]. The twist is similar to that found in the unsubstituted compound, phenyl benzoate (Shibakami \& Sekiya, 1995).

\section{S2. Experimental}

4-Allyl-2-methoxyphenol ( $1 \mathrm{mmol})$, benzoic acid $(1 \mathrm{mmol})$, diethylazodicarboxylate $(2 \mathrm{mmol})$ and triphenylphosphine $(2$ mmol) were heated in THF $(10 \mathrm{ml})$ for $2 \mathrm{~h}$. The solid material extracted with dichloromethane. The dichloromethane solution was eluted through a silica gel column by using an $n$-hexane-ethyl acetate $(95: 5 v / v)$ solvent system. Slow evaporation of the solution yielded large colorless crystals.

\section{S3. Refinement}

H-atoms were placed in calculated positions [C-H 0.95 to $0.98 \AA, U_{\text {iso }}(\mathrm{H}) 1.2$ to $\left.1.5 U_{\text {eq }}(\mathrm{C})\right]$ and were included in the refinement in the riding model approximation.

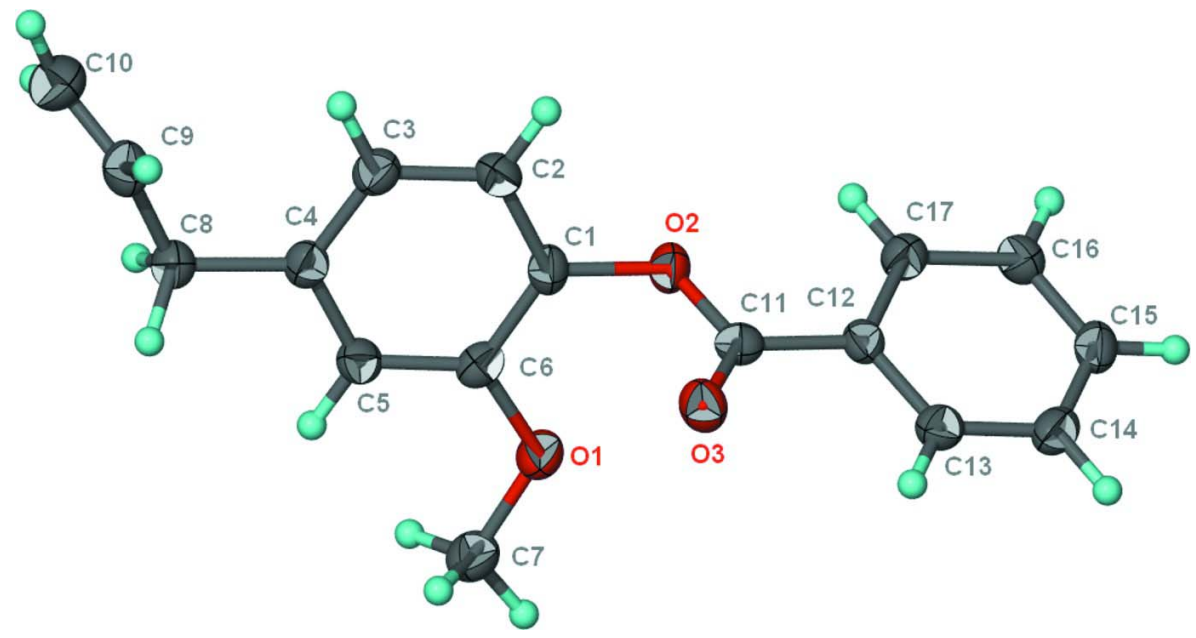

Figure 1

Thermal ellipsoid plot (Barbour, 2001) of $\mathrm{C}_{17} \mathrm{H}_{16} \mathrm{O}_{3}$ at the $70 \%$ probability level; hydrogen atoms are drawn as spheres of arbitrary radius. 
2-Methoxy-4-(prop-2-en-1-yl)phenyl benzoate

Crystal data

$\mathrm{C}_{17} \mathrm{H}_{16} \mathrm{O}_{3}$

$M_{r}=268.30$

Monoclinic, $P 2_{1} / c$

Hall symbol: -P 2ybc

$a=9.9334(6) \AA$

$b=9.5124(5) \AA$

$c=14.9463(9) \AA$

$\beta=103.405(6)^{\circ}$

$V=1373.81(14) \AA^{3}$

$Z=4$

$F(000)=568$

$D_{\mathrm{x}}=1.297 \mathrm{Mg} \mathrm{m}^{-3}$

Mo $K \alpha$ radiation, $\lambda=0.71073 \AA$

Cell parameters from 1957 reflections

$\theta=3.0-27.5^{\circ}$

$\mu=0.09 \mathrm{~mm}^{-1}$

$T=100 \mathrm{~K}$

Prism, colorless

$0.40 \times 0.30 \times 0.20 \mathrm{~mm}$

\section{Data collection}

Agilent SuperNova Dual diffractometer with an Atlas detector

Radiation source: SuperNova (Mo) X-ray

Source

Mirror monochromator

Detector resolution: 10.4041 pixels $\mathrm{mm}^{-1}$

$\omega$ scan

Absorption correction: multi-scan

(CrysAlis PRO; Agilent, 2013)

$T_{\min }=0.966, T_{\max }=0.983$

7024 measured reflections

3168 independent reflections

2242 reflections with $I>2 \sigma(I)$

$R_{\text {int }}=0.041$

$\theta_{\max }=27.6^{\circ}, \theta_{\min }=3.0^{\circ}$

$h=-10 \rightarrow 12$

$k=-9 \rightarrow 12$

$l=-19 \rightarrow 16$

Refinement

Refinement on $F^{2}$

Least-squares matrix: full

$R\left[F^{2}>2 \sigma\left(F^{2}\right)\right]=0.051$

$w R\left(F^{2}\right)=0.138$

$S=1.05$

3168 reflections

181 parameters

0 restraints

Primary atom site location: structure-invariant direct methods

Secondary atom site location: difference Fourier map

Hydrogen site location: inferred from neighbouring sites

$\mathrm{H}$-atom parameters constrained

$w=1 /\left[\sigma^{2}\left(F_{\mathrm{o}}^{2}\right)+(0.0572 P)^{2}+0.1625 P\right]$

where $P=\left(F_{\mathrm{o}}^{2}+2 F_{\mathrm{c}}{ }^{2}\right) / 3$

$(\Delta / \sigma)_{\max }=0.001$

$\Delta \rho_{\max }=0.23 \mathrm{e} \AA^{-3}$

$\Delta \rho_{\min }=-0.29 \mathrm{e}^{-3}$

Fractional atomic coordinates and isotropic or equivalent isotropic displacement parameters $\left(\AA^{2}\right)$

\begin{tabular}{lllll}
\hline & $x$ & $y$ & $z$ & $U_{\text {iso }}^{*} / U_{\text {eq }}$ \\
\hline O1 & $0.40190(12)$ & $0.28310(12)$ & $0.60263(8)$ & $0.0266(3)$ \\
O2 & $0.22747(12)$ & $0.48286(12)$ & $0.63406(8)$ & $0.0241(3)$ \\
O3 & $0.09998(13)$ & $0.28409(12)$ & $0.60909(8)$ & $0.0266(3)$ \\
C1 & $0.25534(17)$ & $0.47175(17)$ & $0.54614(11)$ & $0.0216(4)$ \\
C2 & $0.19517(17)$ & $0.56488(17)$ & $0.47895(12)$ & $0.0232(4)$ \\
H2 & 0.1302 & 0.6319 & 0.4904 & $0.028^{*}$ \\
C3 & $0.22950(17)$ & $0.56121(18)$ & $0.39346(12)$ & $0.0236(4)$ \\
H3 & 0.1877 & 0.6257 & 0.3467 & $0.028^{*}$ \\
C4 & $0.32409(17)$ & $0.46399(17)$ & $0.37666(11)$ & $0.0216(4)$ \\
C5 & $0.38323(17)$ & $0.36863(17)$ & $0.44573(11)$ & $0.0220(4)$ \\
H5 & 0.4472 & 0.3007 & 0.4341 & $0.026^{*}$ \\
C6 & $0.35012(17)$ & $0.37154(17)$ & $0.53098(11)$ & $0.0218(4)$ \\
C7 & $0.48978(19)$ & $0.1729(2)$ & $0.58544(13)$ & $0.0305(4)$
\end{tabular}




$\begin{array}{lllll}\text { H7A } & 0.5215 & 0.1171 & 0.6415 & 0.046^{*} \\ \text { H7B } & 0.5699 & 0.2133 & 0.5669 & 0.046^{*} \\ \text { H7C } & 0.4384 & 0.1124 & 0.5361 & 0.046^{*} \\ \text { C8 } & 0.36682(18) & 0.45729(18) & 0.28547(11) & 0.0241(4) \\ \text { H8A } & 0.4647 & 0.4870 & 0.2957 & 0.029^{*} \\ \text { H8B } & 0.3613 & 0.3583 & 0.2645 & 0.029^{*} \\ \text { C9 } & 0.2825(2) & 0.5455(2) & 0.21071(12) & 0.0300(4) \\ \text { H9 } & 0.1877 & 0.5214 & 0.1888 & 0.036^{*} \\ \text { C10 } & 0.3292(3) & 0.6537(2) & 0.17302(14) & 0.0429(5) \\ \text { H10A } & 0.4234 & 0.6809 & 0.1931 & 0.052^{*} \\ \text { H10B } & 0.2690 & 0.7049 & 0.1255 & 0.052^{*} \\ \text { C11 } & 0.15078(17) & 0.37820(17) & 0.65969(12) & 0.0215(4) \\ \text { C12 } & 0.13802(17) & 0.39478(17) & 0.75630(11) & 0.0206(4) \\ \text { C13 } & 0.08089(17) & 0.28421(18) & 0.79540(12) & 0.0233(4) \\ \text { H13 } & 0.0493 & 0.2026 & 0.7601 & 0.028^{*} \\ \text { C14 } & 0.06989(18) & 0.29291(19) & 0.88614(12) & 0.0264(4) \\ \text { H14 } & 0.0324 & 0.2164 & 0.9133 & 0.032^{*} \\ \text { C15 } & 0.11346(18) & 0.41292(18) & 0.93709(12) & 0.0261(4) \\ \text { H15 } & 0.1064 & 0.4185 & 0.9993 & 0.031^{*} \\ \text { C16 } & 0.16721(18) & 0.52473(18) & 0.89742(12) & 0.0262(4) \\ \text { H16 } & 0.1947 & 0.6079 & 0.9320 & 0.031^{*} \\ \text { C17 } & 0.18109(18) & 0.51586(18) & 0.80734(12) & 0.0233(4) \\ \text { H17 } & 0.2198 & 0.5920 & 0.7806 & 0.028^{*} \\ \end{array}$

Atomic displacement parameters $\left(\AA^{2}\right)$

\begin{tabular}{lllllll}
\hline & $U^{11}$ & $U^{22}$ & $U^{33}$ & $U^{12}$ & $U^{13}$ & $U^{23}$ \\
\hline O1 & $0.0297(7)$ & $0.0284(7)$ & $0.0225(6)$ & $0.0055(6)$ & $0.0076(5)$ & $0.0056(5)$ \\
O2 & $0.0305(7)$ & $0.0244(6)$ & $0.0196(6)$ & $-0.0024(5)$ & $0.0102(5)$ & $-0.0015(5)$ \\
O3 & $0.0295(7)$ & $0.0267(7)$ & $0.0244(7)$ & $-0.0031(6)$ & $0.0082(5)$ & $-0.0049(5)$ \\
C1 & $0.0244(9)$ & $0.0236(9)$ & $0.0183(8)$ & $-0.0042(7)$ & $0.0078(7)$ & $-0.0027(7)$ \\
C2 & $0.0261(9)$ & $0.0196(8)$ & $0.0255(9)$ & $0.0013(7)$ & $0.0089(7)$ & $-0.0018(7)$ \\
C3 & $0.0268(9)$ & $0.0228(8)$ & $0.0210(9)$ & $-0.0002(8)$ & $0.0048(7)$ & $0.0019(7)$ \\
C4 & $0.0247(9)$ & $0.0211(8)$ & $0.0193(8)$ & $-0.0039(7)$ & $0.0054(7)$ & $-0.0022(7)$ \\
C5 & $0.0208(9)$ & $0.0215(8)$ & $0.0251(9)$ & $0.0002(7)$ & $0.0079(7)$ & $0.0000(7)$ \\
C6 & $0.0219(8)$ & $0.0215(8)$ & $0.0212(8)$ & $-0.0028(7)$ & $0.0032(7)$ & $0.0027(7)$ \\
C7 & $0.0315(10)$ & $0.0299(10)$ & $0.0310(10)$ & $0.0054(9)$ & $0.0094(8)$ & $0.0073(8)$ \\
C8 & $0.0283(9)$ & $0.0243(9)$ & $0.0210(9)$ & $0.0010(8)$ & $0.0081(7)$ & $0.0000(7)$ \\
C9 & $0.0360(10)$ & $0.0338(10)$ & $0.0212(9)$ & $0.0042(9)$ & $0.0087(8)$ & $-0.0002(8)$ \\
C10 & $0.0651(15)$ & $0.0344(11)$ & $0.0321(11)$ & $0.0090(11)$ & $0.0171(11)$ & $0.0076(9)$ \\
C11 & $0.0210(8)$ & $0.0197(8)$ & $0.0232(9)$ & $0.0028(7)$ & $0.0039(7)$ & $0.0016(7)$ \\
C12 & $0.0214(8)$ & $0.0223(8)$ & $0.0189(8)$ & $0.0045(7)$ & $0.0060(6)$ & $0.0010(7)$ \\
C13 & $0.0230(9)$ & $0.0218(9)$ & $0.0255(9)$ & $0.0016(7)$ & $0.0066(7)$ & $0.0010(7)$ \\
C14 & $0.0263(9)$ & $0.0260(9)$ & $0.0291(10)$ & $0.0026(8)$ & $0.0109(7)$ & $0.0070(8)$ \\
C15 & $0.0285(9)$ & $0.0315(10)$ & $0.0199(9)$ & $0.0070(8)$ & $0.0087(7)$ & $0.0033(8)$ \\
C16 & $0.0319(10)$ & $0.0243(9)$ & $0.0232(9)$ & $0.0011(8)$ & $0.0079(8)$ & $-0.0030(7)$ \\
C17 & $0.0254(9)$ & $0.0221(9)$ & $0.0230(9)$ & $-0.0012(7)$ & $0.0068(7)$ & $0.0008(7)$ \\
& & & & & &
\end{tabular}


Geometric parameters $\left(\AA,{ }^{\circ}\right)$

\begin{tabular}{|c|c|c|c|}
\hline $\mathrm{O} 1-\mathrm{C} 6$ & $1.3652(19)$ & $\mathrm{C} 8-\mathrm{H} 8 \mathrm{~A}$ & 0.9900 \\
\hline $\mathrm{O} 1-\mathrm{C} 7$ & $1.425(2)$ & $\mathrm{C} 8-\mathrm{H} 8 \mathrm{~B}$ & 0.9900 \\
\hline $\mathrm{O} 2-\mathrm{C} 11$ & $1.361(2)$ & $\mathrm{C} 9-\mathrm{C} 10$ & $1.309(3)$ \\
\hline $\mathrm{O} 2-\mathrm{C} 1$ & $1.4084(19)$ & $\mathrm{C} 9-\mathrm{H} 9$ & 0.9500 \\
\hline $\mathrm{O} 3-\mathrm{C} 11$ & $1.205(2)$ & $\mathrm{C} 10-\mathrm{H} 10 \mathrm{~A}$ & 0.9500 \\
\hline $\mathrm{C} 1-\mathrm{C} 2$ & $1.368(2)$ & $\mathrm{C} 10-\mathrm{H} 10 \mathrm{~B}$ & 0.9500 \\
\hline $\mathrm{C} 1-\mathrm{C} 6$ & $1.395(2)$ & $\mathrm{C} 11-\mathrm{C} 12$ & $1.487(2)$ \\
\hline $\mathrm{C} 2-\mathrm{C} 3$ & $1.397(2)$ & $\mathrm{C} 12-\mathrm{C} 13$ & $1.387(2)$ \\
\hline $\mathrm{C} 2-\mathrm{H} 2$ & 0.9500 & $\mathrm{C} 12-\mathrm{C} 17$ & $1.393(2)$ \\
\hline $\mathrm{C} 3-\mathrm{C} 4$ & $1.382(2)$ & $\mathrm{C} 13-\mathrm{C} 14$ & $1.388(2)$ \\
\hline $\mathrm{C} 3-\mathrm{H} 3$ & 0.9500 & $\mathrm{C} 13-\mathrm{H} 13$ & 0.9500 \\
\hline $\mathrm{C} 4-\mathrm{C} 5$ & $1.397(2)$ & $\mathrm{C} 14-\mathrm{C} 15$ & $1.385(2)$ \\
\hline $\mathrm{C} 4-\mathrm{C} 8$ & $1.520(2)$ & $\mathrm{C} 14-\mathrm{H} 14$ & 0.9500 \\
\hline $\mathrm{C} 5-\mathrm{C} 6$ & $1.388(2)$ & $\mathrm{C} 15-\mathrm{C} 16$ & $1.384(2)$ \\
\hline $\mathrm{C} 5-\mathrm{H} 5$ & 0.9500 & $\mathrm{C} 15-\mathrm{H} 15$ & 0.9500 \\
\hline $\mathrm{C} 7-\mathrm{H} 7 \mathrm{~A}$ & 0.9800 & $\mathrm{C} 16-\mathrm{C} 17$ & $1.387(2)$ \\
\hline C7-H7B & 0.9800 & $\mathrm{C} 16-\mathrm{H} 16$ & 0.9500 \\
\hline $\mathrm{C} 7-\mathrm{H} 7 \mathrm{C}$ & 0.9800 & C17-H17 & 0.9500 \\
\hline $\mathrm{C} 8-\mathrm{C} 9$ & $1.490(2)$ & & \\
\hline $\mathrm{C} 6-\mathrm{O} 1-\mathrm{C} 7$ & $116.55(13)$ & $\mathrm{C} 4-\mathrm{C} 8-\mathrm{H} 8 \mathrm{~B}$ & 108.5 \\
\hline $\mathrm{C} 11-\mathrm{O} 2-\mathrm{C} 1$ & $116.84(13)$ & $\mathrm{H} 8 \mathrm{~A}-\mathrm{C} 8-\mathrm{H} 8 \mathrm{~B}$ & 107.5 \\
\hline $\mathrm{C} 2-\mathrm{C} 1-\mathrm{C} 6$ & $121.32(15)$ & $\mathrm{C} 10-\mathrm{C} 9-\mathrm{C} 8$ & $124.85(19)$ \\
\hline $\mathrm{C} 2-\mathrm{C} 1-\mathrm{O} 2$ & $119.29(15)$ & $\mathrm{C} 10-\mathrm{C} 9-\mathrm{H} 9$ & 117.6 \\
\hline $\mathrm{C} 6-\mathrm{C} 1-\mathrm{O} 2$ & $119.27(14)$ & $\mathrm{C} 8-\mathrm{C} 9-\mathrm{H} 9$ & 117.6 \\
\hline $\mathrm{C} 1-\mathrm{C} 2-\mathrm{C} 3$ & $119.82(16)$ & $\mathrm{C} 9-\mathrm{C} 10-\mathrm{H} 10 \mathrm{~A}$ & 120.0 \\
\hline $\mathrm{C} 1-\mathrm{C} 2-\mathrm{H} 2$ & 120.1 & $\mathrm{C} 9-\mathrm{C} 10-\mathrm{H} 10 \mathrm{~B}$ & 120.0 \\
\hline $\mathrm{C} 3-\mathrm{C} 2-\mathrm{H} 2$ & 120.1 & $\mathrm{H} 10 \mathrm{~A}-\mathrm{C} 10-\mathrm{H} 10 \mathrm{~B}$ & 120.0 \\
\hline $\mathrm{C} 4-\mathrm{C} 3-\mathrm{C} 2$ & $120.21(16)$ & $\mathrm{O} 3-\mathrm{C} 11-\mathrm{O} 2$ & $123.18(15)$ \\
\hline $\mathrm{C} 4-\mathrm{C} 3-\mathrm{H} 3$ & 119.9 & $\mathrm{O} 3-\mathrm{C} 11-\mathrm{C} 12$ & $124.81(16)$ \\
\hline $\mathrm{C} 2-\mathrm{C} 3-\mathrm{H} 3$ & 119.9 & $\mathrm{O} 2-\mathrm{C} 11-\mathrm{C} 12$ & $112.02(14)$ \\
\hline $\mathrm{C} 3-\mathrm{C} 4-\mathrm{C} 5$ & $119.15(15)$ & $\mathrm{C} 13-\mathrm{C} 12-\mathrm{C} 17$ & $120.00(15)$ \\
\hline $\mathrm{C} 3-\mathrm{C} 4-\mathrm{C} 8$ & $122.27(15)$ & $\mathrm{C} 13-\mathrm{C} 12-\mathrm{C} 11$ & $117.65(15)$ \\
\hline $\mathrm{C} 5-\mathrm{C} 4-\mathrm{C} 8$ & $118.58(15)$ & $\mathrm{C} 17-\mathrm{C} 12-\mathrm{C} 11$ & $122.34(15)$ \\
\hline $\mathrm{C} 6-\mathrm{C} 5-\mathrm{C} 4$ & $121.12(16)$ & $\mathrm{C} 12-\mathrm{C} 13-\mathrm{C} 14$ & $119.94(16)$ \\
\hline $\mathrm{C} 6-\mathrm{C} 5-\mathrm{H} 5$ & 119.4 & $\mathrm{C} 12-\mathrm{C} 13-\mathrm{H} 13$ & 120.0 \\
\hline $\mathrm{C} 4-\mathrm{C} 5-\mathrm{H} 5$ & 119.4 & $\mathrm{C} 14-\mathrm{C} 13-\mathrm{H} 13$ & 120.0 \\
\hline $\mathrm{O} 1-\mathrm{C} 6-\mathrm{C} 5$ & $125.55(15)$ & $\mathrm{C} 15-\mathrm{C} 14-\mathrm{C} 13$ & $120.07(16)$ \\
\hline $\mathrm{O} 1-\mathrm{C} 6-\mathrm{C} 1$ & $116.07(14)$ & $\mathrm{C} 15-\mathrm{C} 14-\mathrm{H} 14$ & 120.0 \\
\hline $\mathrm{C} 5-\mathrm{C} 6-\mathrm{C} 1$ & $118.37(15)$ & $\mathrm{C} 13-\mathrm{C} 14-\mathrm{H} 14$ & 120.0 \\
\hline $\mathrm{O} 1-\mathrm{C} 7-\mathrm{H} 7 \mathrm{~A}$ & 109.5 & $\mathrm{C} 16-\mathrm{C} 15-\mathrm{C} 14$ & $120.03(16)$ \\
\hline $\mathrm{O} 1-\mathrm{C} 7-\mathrm{H} 7 \mathrm{~B}$ & 109.5 & $\mathrm{C} 16-\mathrm{C} 15-\mathrm{H} 15$ & 120.0 \\
\hline $\mathrm{H} 7 \mathrm{~A}-\mathrm{C} 7-\mathrm{H} 7 \mathrm{~B}$ & 109.5 & $\mathrm{C} 14-\mathrm{C} 15-\mathrm{H} 15$ & 120.0 \\
\hline $\mathrm{O} 1-\mathrm{C} 7-\mathrm{H} 7 \mathrm{C}$ & 109.5 & $\mathrm{C} 15-\mathrm{C} 16-\mathrm{C} 17$ & $120.27(16)$ \\
\hline $\mathrm{H} 7 \mathrm{~A}-\mathrm{C} 7-\mathrm{H} 7 \mathrm{C}$ & 109.5 & $\mathrm{C} 15-\mathrm{C} 16-\mathrm{H} 16$ & 119.9 \\
\hline $\mathrm{H} 7 \mathrm{~B}-\mathrm{C} 7-\mathrm{H} 7 \mathrm{C}$ & 109.5 & $\mathrm{C} 17-\mathrm{C} 16-\mathrm{H} 16$ & 119.9 \\
\hline
\end{tabular}




$\begin{array}{llll}\mathrm{C} 9-\mathrm{C} 8-\mathrm{C} 4 & 115.06(14) & \mathrm{C} 16-\mathrm{C} 17-\mathrm{C} 12 & 119.65(16) \\ \mathrm{C} 9-\mathrm{C} 8-\mathrm{H} 8 \mathrm{~A} & 108.5 & \mathrm{C} 16-\mathrm{C} 17-\mathrm{H} 17 & 120.2 \\ \mathrm{C} 4-\mathrm{C} 8-\mathrm{H} 8 \mathrm{~A} & 108.5 & \mathrm{C} 12-\mathrm{C} 17-\mathrm{H} 17 & \\ \mathrm{C} 9-\mathrm{C} 8-\mathrm{H} 8 \mathrm{~B} & 108.5 & & \\ \mathrm{C} 11-\mathrm{O} 2-\mathrm{C} 1-\mathrm{C} 2 & & \mathrm{C} 3-\mathrm{C} 4-\mathrm{C} 8-\mathrm{C} 9 & -10.0(2) \\ \mathrm{C} 11-\mathrm{O} 2-\mathrm{C} 1-\mathrm{C} 6 & -72.7(2) & \mathrm{C} 5-\mathrm{C} 4-\mathrm{C} 8-\mathrm{C} 9 & 170.21(15) \\ \mathrm{C} 6-\mathrm{C} 1-\mathrm{C} 2-\mathrm{C} 3 & -0.5(3) & \mathrm{C} 4-\mathrm{C} 8-\mathrm{C} 9-\mathrm{C} 10 & 114.8(2) \\ \mathrm{O} 2-\mathrm{C} 1-\mathrm{C} 2-\mathrm{C} 3 & 175.52(14) & \mathrm{C} 1-\mathrm{O} 2-\mathrm{C} 11-\mathrm{O} 3 & -4.8(2) \\ \mathrm{C} 1-\mathrm{C} 2-\mathrm{C} 3-\mathrm{C} 4 & -0.1(3) & \mathrm{C} 1-\mathrm{O} 2-\mathrm{C} 11-\mathrm{C} 12 & 175.22(13) \\ \mathrm{C} 2-\mathrm{C} 3-\mathrm{C} 4-\mathrm{C} 5 & 0.8(2) & \mathrm{O} 3-\mathrm{C} 11-\mathrm{C} 12-\mathrm{C} 13 & 9.7(2) \\ \mathrm{C} 2-\mathrm{C} 3-\mathrm{C} 4-\mathrm{C} 8 & -178.92(15) & \mathrm{O} 2-\mathrm{C} 11-\mathrm{C} 12-\mathrm{C} 13 & -170.29(14) \\ \mathrm{C} 3-\mathrm{C} 4-\mathrm{C} 5-\mathrm{C} 6 & -1.0(2) & \mathrm{O} 3-\mathrm{C} 11-\mathrm{C} 12-\mathrm{C} 17 & -170.28(16) \\ \mathrm{C} 8-\mathrm{C} 4-\mathrm{C} 5-\mathrm{C} 6 & 178.77(15) & \mathrm{O} 2-\mathrm{C} 11-\mathrm{C} 12-\mathrm{C} 17 & 9.7(2) \\ \mathrm{C} 7-\mathrm{O} 1-\mathrm{C} 6-\mathrm{C} 5 & -4.6(2) & \mathrm{C} 17-\mathrm{C} 12-\mathrm{C} 13-\mathrm{C} 14 & -1.6(3) \\ \mathrm{C} 7-\mathrm{O} 1-\mathrm{C} 6-\mathrm{C} 1 & 174.89(15) & \mathrm{C} 11-\mathrm{C} 12-\mathrm{C} 13-\mathrm{C} 14 & 178.36(15) \\ \mathrm{C} 4-\mathrm{C} 5-\mathrm{C} 6-\mathrm{O} 1 & 179.88(15) & \mathrm{C} 12-\mathrm{C} 13-\mathrm{C} 14-\mathrm{C} 15 & 1.2(3) \\ \mathrm{C} 4-\mathrm{C} 5-\mathrm{C} 6-\mathrm{C} 1 & 0.4(2) & \mathrm{C} 13-\mathrm{C} 14-\mathrm{C} 15-\mathrm{C} 16 & 0.4(3) \\ \mathrm{C} 2-\mathrm{C} 1-\mathrm{C} 6-\mathrm{O} 1 & -179.15(15) & \mathrm{C} 14-\mathrm{C} 15-\mathrm{C} 16-\mathrm{C} 17 & -1.7(3) \\ \mathrm{O} 2-\mathrm{C} 1-\mathrm{C} 6-\mathrm{O} 1 & 4.8(2) & \mathrm{C} 15-\mathrm{C} 16-\mathrm{C} 17-\mathrm{C} 12 & 1.3(3) \\ \mathrm{C} 2-\mathrm{C} 1-\mathrm{C} 6-\mathrm{C} 5 & 0.4(2) & \mathrm{C} 13-\mathrm{C} 12-\mathrm{C} 17-\mathrm{C} 16 & 0.4(3) \\ \mathrm{O} 2-\mathrm{C} 1-\mathrm{C} 6-\mathrm{C} 5 & -175.68(14) & \mathrm{C} 11-\mathrm{C} 12-\mathrm{C} 17-\mathrm{C} 16 & -179.62(15)\end{array}$

Hydrogen-bond geometry $\left(\AA,{ }^{\circ}\right)$

\begin{tabular}{lllll}
\hline$D-\mathrm{H} \cdots A$ & $D-\mathrm{H}$ & $\mathrm{H} \cdots A$ & $D \cdots A$ & $D-\mathrm{H} \cdots A$ \\
\hline $\mathrm{C} 2-\mathrm{H} 2 \cdots \mathrm{O}^{\mathrm{i}}$ & 0.95 & 2.55 & $3.256(2)$ & 131 \\
$\mathrm{C} 15-\mathrm{H} 15 \cdots \mathrm{O} 3^{\mathrm{ii}}$ & 0.95 & 2.54 & $3.209(2)$ & 128 \\
\hline
\end{tabular}

Symmetry codes: (i) $-x,-y+1,-z+1$; (ii) $x,-y+1 / 2, z+1 / 2$. 